\title{
Locally Controlled Diffusive Release of Bone Morphogenetic Protein-2 Using Micropatterned Gelatin Methacrylate Hydrogel Carriers
}

\author{
Myong-Hee $\mathrm{Yi}^{1}$, Ji-Eun Lee ${ }^{2}$, Chang-Beom Kim ${ }^{3}$, Keun-Woo Lee (iD ${ }^{1, *}$ \& Kwang-Ho Lee (iD ${ }^{4, *}$
}

Received: 26 May, 2020 / Accepted: 7 August, 2020 / Published online: 18 November, 2020

(C) The Korean BioChip Society and Springer 2020

\begin{abstract}
In this work, a novel and simple bone morphogenetic protein (BMP)-2 carrier is developed, which enables localized and controlled release of BMP-2 and facilitates bone regeneration. BMP-2 is localized in the gelatin methacrylate (GelMA) micropatterns on hydrophilic semi-permeable membrane (SNM), and its controlled release is regulated by the concentration of GelMA hydrogel and BMP-2. The controlled release of BMP-2 is verified using computational analysis and quantified using fluorescein isothiocyanate-bovine serum albumin (FITC-BSA) diffusion model. The osteogenic differentiation of osteosarcoma MG-63 cells is manipulated by localized and controlled BMP-2 release. The calcium deposits are significantly higher and the actin skeletal networks are denser in MG-63 cells cultured in the BMP-2immobilized GelMA micropattern than in the absence of BMP-2. The proposed BMP-2 carrier is expected to not only act as a barrier membrane that can prevent invasion of connective tissue during bone regeneration, but also as a carrier capable of localiz-
\end{abstract}

\footnotetext{
${ }^{1}$ Department of Prosthodontics, College of Dentistry, Yonsei University, Seoul 03722, Republic of Korea

${ }^{2}$ Graduate Program of Advanced Functional Materials and Devices Development, Kangwon National University, Chuncheon, 24341, Republic of Korea

${ }^{3}$ Intelligent Robot Research Team, Electronics and Telecommunications Research Institute, Daejeon 34129, Republic of Korea

${ }^{4}$ Division of Mechanical \& Biomedical, Mechatronics and Materials Science and Engineering, Chuncheon, Kangwon National University, 24341, Republic of Korea

*Correspondence and requests for materials should be addressed to

K-W. Lee $(\bowtie$ kwlee@yuhs.ac) and

K-H. Lee ( $₫$ khmhlee@kangwon.ac.kr)
}

ing and controlling the release of BMP-2 due to GelMA micropatterning on SNM. This approach can be extensively applied to tissue engineering, including the localization and encapsulation of cells or drugs.

Keywords: Bone generation, Gelatin-methacrylate, Localized release, Controlled release, Bone morphogenetic protein-2

\section{Introduction}

Bone regeneration is the coordinated response of the body to injured or damaged bones ${ }^{1-3}$. This is physiologically complicated and involves unknown signaling pathways and processes, which are regulated by different cells, cytokines, and growth factors ${ }^{4-6}$. Autologous bone grafting is still the gold standard for bone regeneration treatment ${ }^{7-9}$. Despite the obvious advantages of immune compatibility and excellent osteoconductive, osteoinductive, and osteogenic properties, the application of bone autografting is limited by the dearth of donor tissue supply and risk of donor site complications. Similarly, the use of allografts and xenografts has been constrained by more serious problems such as immune responses or infections ${ }^{10,11}$. Although these treatments have significantly contributed to bone regeneration, they require complicated, appropriate, and reliable manipulations for successful bone regeneration ${ }^{12}$. To address these problems, comprehensive biomaterial-based substitutes are being used for bone regeneration with autograft, allograft, 
and xenograft procedures ${ }^{13-16}$. Recently, groundbreaking research in bone regenerative medicine has suggested the use of a combination of biomaterial-based bone substitutes and cells ${ }^{17}$. These attempts have revealed new possibilities for circumventing the shortcomings of only cell-based therapies. The major limitations of the current bone substitutes include lack of properties that support complex cell-microenvironment interactions, difficulty in generating bone tissue remodeling due to cell necrosis, and loss of function within the damaged bone tissue ${ }^{18}$. Therefore, regulation of osteogenic differentiation is one of the crucial challenges in bone regenerative medicine for successful bone regeneration therapy. One emerging approach involves the use of bone morphogenetic proteins (BMPs), which are members of the transforming growth factor-beta (TGF- $\beta$ ) superfamily of proteins. BMPs play a pivotal role in the regulation of bone induction and repair as growth factors, cytokines, and metabologens ${ }^{19-21}$. In addition, BMPs play an important role in cell-cell interactions, such as autocrine or paracrine interactions, by associating with cell surface BMP receptors as homo or heterodimers, which affect various cell types. BMPs are stored in the bone matrix and are used by osteogenic cells for bone reconstruction or remodeling 22 . In terms of BMP pharmacokinetics, the microenvironmental conditions, BMP release rate, concentration, and delivery method, and carrier materials and surface conditions are known to be critical elements affecting bone regeneration. However, a quantitative delivery or transport system for BMPs is required for effectively using BMPs for enhancement of bone regeneration. Among the BMPs, BMP-2 has been established as the osteogenic BMP based on its dominant bone-inducing activity. The conventional BMP-2 delivery systems involve combinations of biocompatible materials as carriers (i.e., collagen, hyaluronic acid, poly (ethylene glycol) diacrylate, and gelatin) ${ }^{23,24}$. Although collagen has been approved as the carrier for BMP-2 by the Food and Drug Administration (FDA) of USA, it is known that BMP-2 has low binding affinity for collagen ${ }^{12,25}$. Furthermore, collagen-encapsulated BMP-2 shows uncontrolled and unpredictable release, which can cause side effects at ectopic sites. To address these issues, various approaches have been developed, which include methods for BMP-2 loading, carriers that maintain BMP-2 in a stable state, and biomaterials that ensure controlled and predictably localized release of BMP-2 ${ }^{26-29}$. To use BMP-2 in a delivery system, the physiochemical problems, as well as the biological barriers due to the poor affinity of BMP-2 with carriers, and the difficulty in controlling the BMP release kinetics have to be suitably resolved. Currently, biocompatible polymers such as polylactic acid (PLA), chitosan, gelatin, sodium alginate, poly( $\varepsilon$-caprolactone) (PCL), and poly(lactideco-glycolide) (PLGA) are being used for creating a BMP-2 delivery system, and engineering technologies (micro-/nanotechnology) are being investigated as tools that can provide precise and targeted delivery and/or controlled release of a drug or therapeutic substance. Lately, the micropatterned gelatin array worked as the 3D platform for guiding the formation of physiological morphologies of osteocytes and odontoblasts ${ }^{30}$. Also, the microstructural pattern promoted osteoblast cells growth, proliferation, osteogenic differentiation, mineralization, and bone formation ${ }^{31}$. These approaches should include methods for targeted and reliable drug delivery, controlled drug release, dose titration, and even drug monitoring ${ }^{32}$. The emerging micro- and nanotechnology-based approaches enable control over delivery owing to the ability to manipulate the size and the shape of the carrier in simple, cost-effective, and reliable manner. The purpose of the present study is to develop a novel BMP -2 carrier system for localized and controlled release of BMP-2 using GelMA hydrogel micropatterns on a $\mathrm{SNM}^{33,34}$. The immobilization of BMP-2 on the SNM within the GelMA hydrogel micropatterns may enable its use as a BMP-2 carrier and barrier-membrane for bone regeneration in the clinic. The developed SNM and GelMA hydrogel-based BMP-2 carrier system can be used to regulate the precise delivery of various bone regenerative medicines.

\section{Results and Discussion}

\section{Hydrophilic PU-PF SNM}

The entire process of GelMA hydrogel micropatterning on SNM is shown in Figure 1. In the preliminary test with hydrophobic polyurethane (PU)-based SNM as the substrate, the GelMA solution was partially cross-linked and did not attach onto the SNM (Figure S1, Supporting Information). This indicated that for the GelMA hydrogel to cross-link and adhere onto the SNM, the GelMA solution has to be sufficiently absorbed between the SNM prior to crosslinking. However, the GelMA solution did not completely penetrate the interstitial space of the SNM because of its strong hydrophobicity in pure PU SNM. Hence, we attempted to improve the wettability of pure PU SNM using polyethylene oxide (PEO)-based Pluronic 


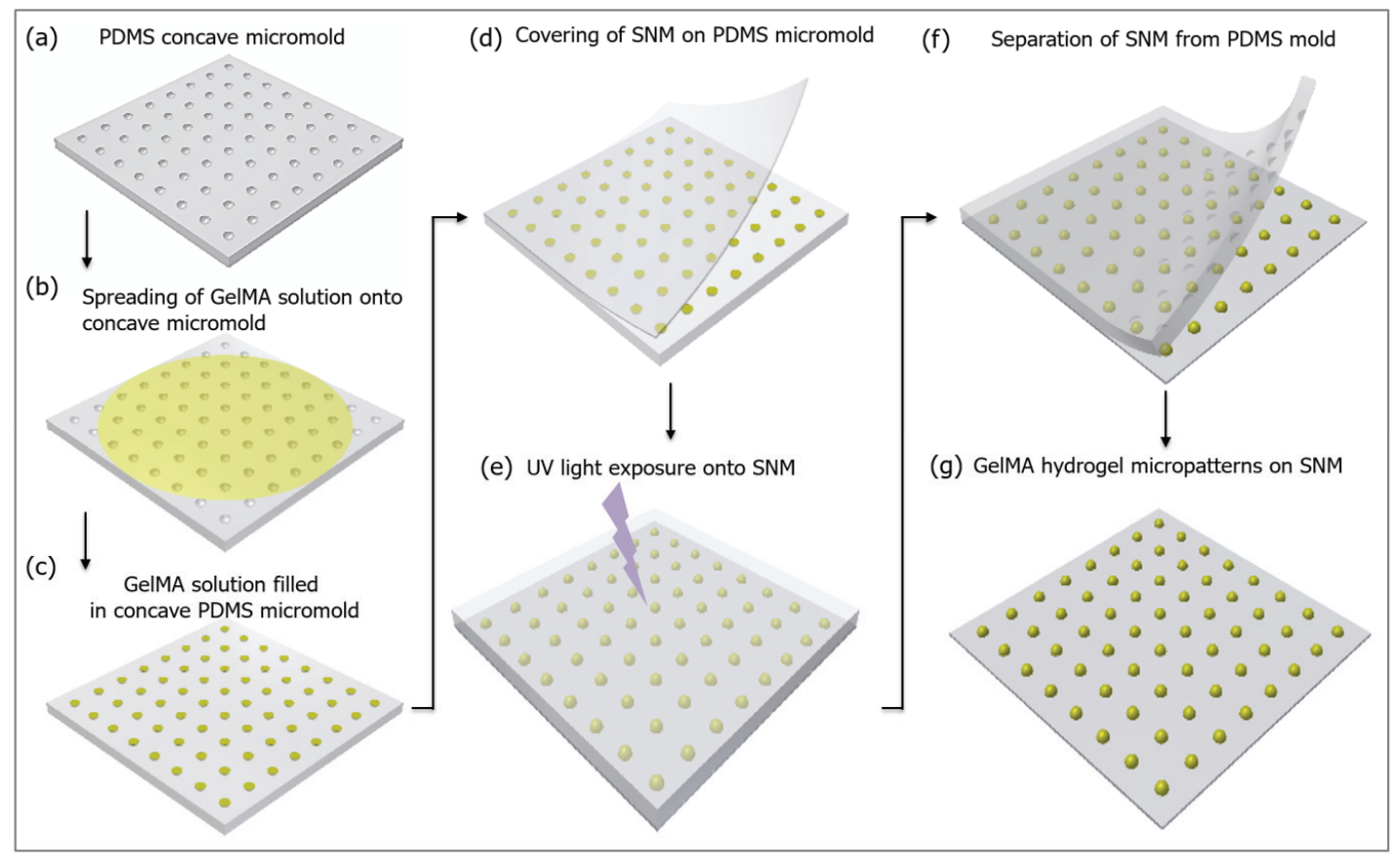

Figure 1. Formation of GelMA hydrogel micropatterns on the semi-permeable nanoporous membrane; (a)-(c) filling of GelMA solution into the PDMS concave micromold, (d)-(e) UV light exposure of the GelMA solution for crosslinking with the mold through the covered membrane, and (f) - (g) GelMA hydrogel micropatterns on the membrane after separation of the mold.
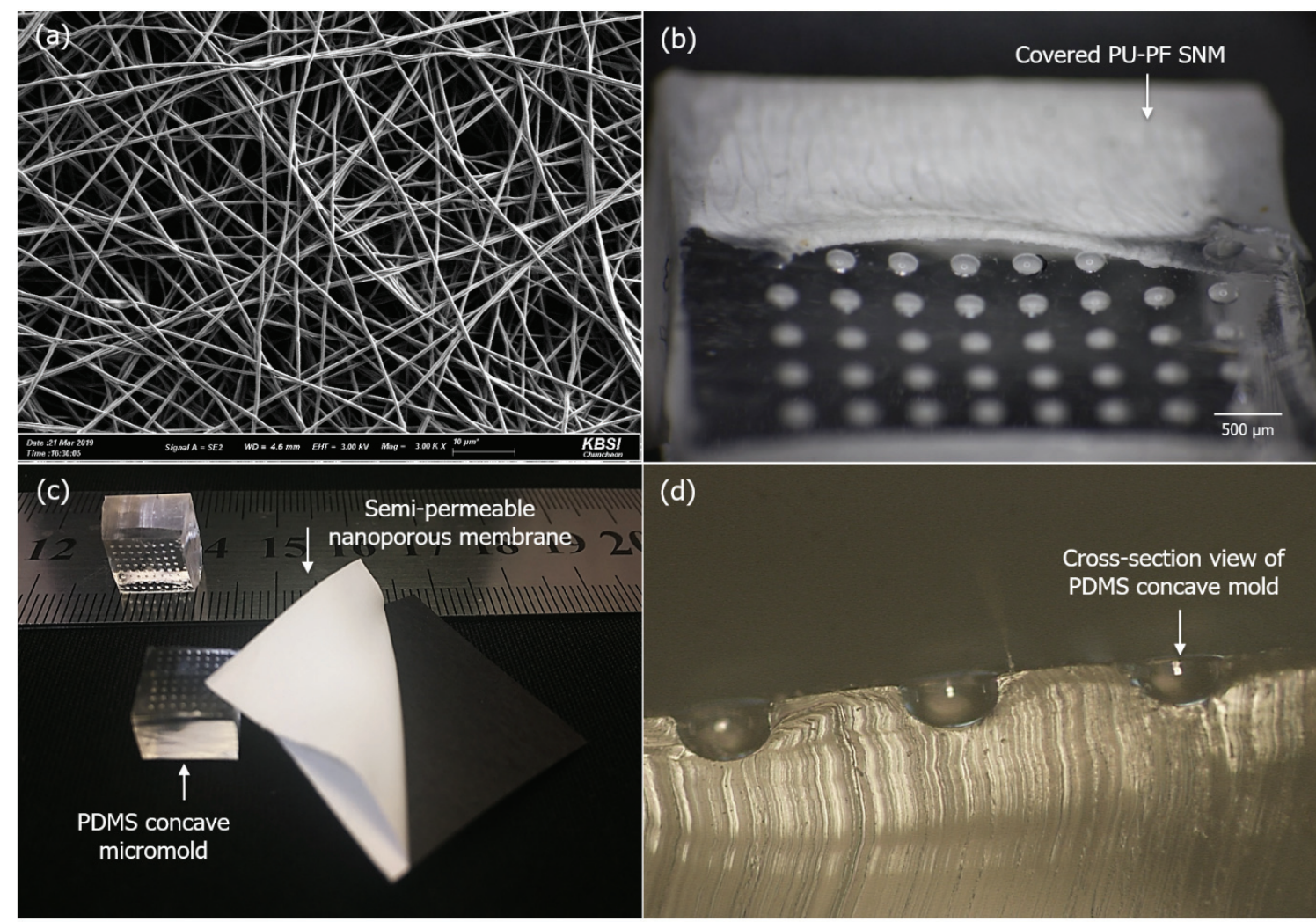

Figure 2. (a) SEM image of electrospun PU-PF SNM, (b) covered PU-PF SNM onto PDMS concave mold, (c) fabricated PDMS concave micromold, and (d) cross-section view of PDMS concave mold. 
F-127 (PF) blending. The novel hydrophilic PU-PF SNM with a uniform nanofiber diameter was successfully produced without droplets, as observed using scanning electron microscopy (Figure 2a). The diameter of the randomly oriented average nanofiber ranged from $200 \mathrm{~nm}$ to $500 \mathrm{~nm}$, and inter-fiber pores were uniformly generated throughout the PU-PF SNM. In terms of altered surface wettability, the contact angle recorded on the PU SNM surface was $82.89 \pm$ $1.3^{\circ}$; however, in PU-PF SNM, the liquid droplets spread completely on the surface, corresponding to a contact angle of $0^{\circ}$ (Figure S2) due to extension of PEO chains, as reported previously ${ }^{35}$. The attenuated total reflection Fourier-transform infrared (ATRFTIR) spectroscopy analysis revealed quantitative information regarding the blended PF. A C-O bond appeared at $1103^{-1}$ in the hydrophilic PU-PF SNM, which increased due to the presence of PF. These results show that semipermeable physical properties with high porosity are sufficient for transmitting ultra-violet (UV) for crosslinking of GelMA hydrogels (Figure S3a,b, Supporting Information). The synthesized GelMA hydrogel was confirmed using an ATRFTIR spectra (Figure S3c, Supporting Information). The identified methacrylation spectra of gelatin were similar to that reported previously ${ }^{36}$, except for a broad peak for the hydroxyl group at $3,500 \mathrm{~cm}^{-1}$ and subdued peaks at $1,000 \sim 1,700 \mathrm{~cm}^{-1}$ for the GelMA hydrogel, which can be attributed to the $-\mathrm{OH}$ group, indicating that both peaks originated from the $\mathrm{C}=\mathrm{C}$ bonds ${ }^{37}$.

\section{Micropatterning for GelMA Hydrogel}

Based on the polydimethylsiloxane (PDMS) concave mold, the single pattern size of the GelMA hydrogel was $250 \mu \mathrm{m}$, although the actual average size was approximately $270 \mu \mathrm{m}$ on the image due to the swelling of the GelMA hydrogel. As shown in Figure 3a, the GelMA hydrogel showed successfully hemispheric cross-linking on flat smooth cover glass surface, as well as on porous mesh-like PU-PF SNM. In Figure $3 b, c$, the addition of fluorescent labeled FITC-BSA and trypan blue confirmed that the micropatterns were clearly formed only in the targeted region, indicating that crosslinking and patterning were possible even if suitable materials were added to the GelMA hydrogel. Figure 3d,e shows the Zstack image observed using a confocal laser microscope. When the fixed micro-beads were monitored while moving along the $\mathrm{Z}$-axis, we observed that the fluorescent beads were evenly dispersed in the pat- tern. These results involve that even if BMP-2 is immobilized and patterned in the GelMA hydrogel, it can be immobilized stably and homogeneously therein. The GelMA hydrogel was intact even after pressing it with the tip of an ink pen (Figure 4). The compressive strength was determined from both the crosslinking density of the GelMA hydrogel and the adhesion between the GelMA hydrogel and SNM. The compressive strengths were $1.064 \mathrm{MPa}$ at the lowest concentration $(2.5 \% \mathrm{w} / \mathrm{v}), 4.556 \mathrm{MPa}$ at the middle concentration $(5 \% \mathrm{w} / \mathrm{v})$, and $6.504 \mathrm{MPa}$ at the highest concentration $(10 \% \mathrm{w} / \mathrm{v})$. If the compressive strength is weak, the surrounding environment and hydrostatic pressure may crack the GelMA hydrogel pattern. On the contrary, if the strength is too high, the slow release of BMP-2 can be suppressed due to the high density of GelMA; however, the value measured in this experiment has been analyzed previously and found to be suitable for controlled BMP-2 release without affecting the GelMA pattern as a viscoelastic material ${ }^{38-40}$. Even when the same specimen was pulled horizontally lengthwise and diagonally, the patterned GelMA hydrogel remained reliably attached and did not separate from the SNM. These results showed that GelMA hydrogels are strongly attached, cross-linked, and patterned on the surface of the three-dimensional network-based SNM using the novel method proposed in the study.

\section{Cross-linking for the BMP-2 Encapsulated GelMA Micropatterning on SNM}

As predicted, the hemisphere radius tended to decrease with increase in the concentration of the GelMA hydrogel and BMP-2, and accurate patterning was formed as the hydrophilicity of SNM increased. Although the increase in UV irradiation intensity and exposure time was increased at high concentration of the GelMA hydrogel, the pattern completeness could not be improved significantly. When hydrophobic SNM was used, it remained in the PDMS mold without adhesion to SNM at all GelMA hydrogel concentrations (Figure S1, Supporting Information). However, the adhesion pattern was partially observed in the hydrophilic SNM, although the concentration of the GelMA hydrogel was increased. To confirm the possibility of loading of BMP-2 in the GelMA hydrogel, fluorescent beads and FITC-BSA were used instead of BMP-2 (which is transparent and difficult to observe), and pattern formation was observed with increasing GelMA hydrogel concentration. At the lowest concentration, the FITC-BSA distribution was 

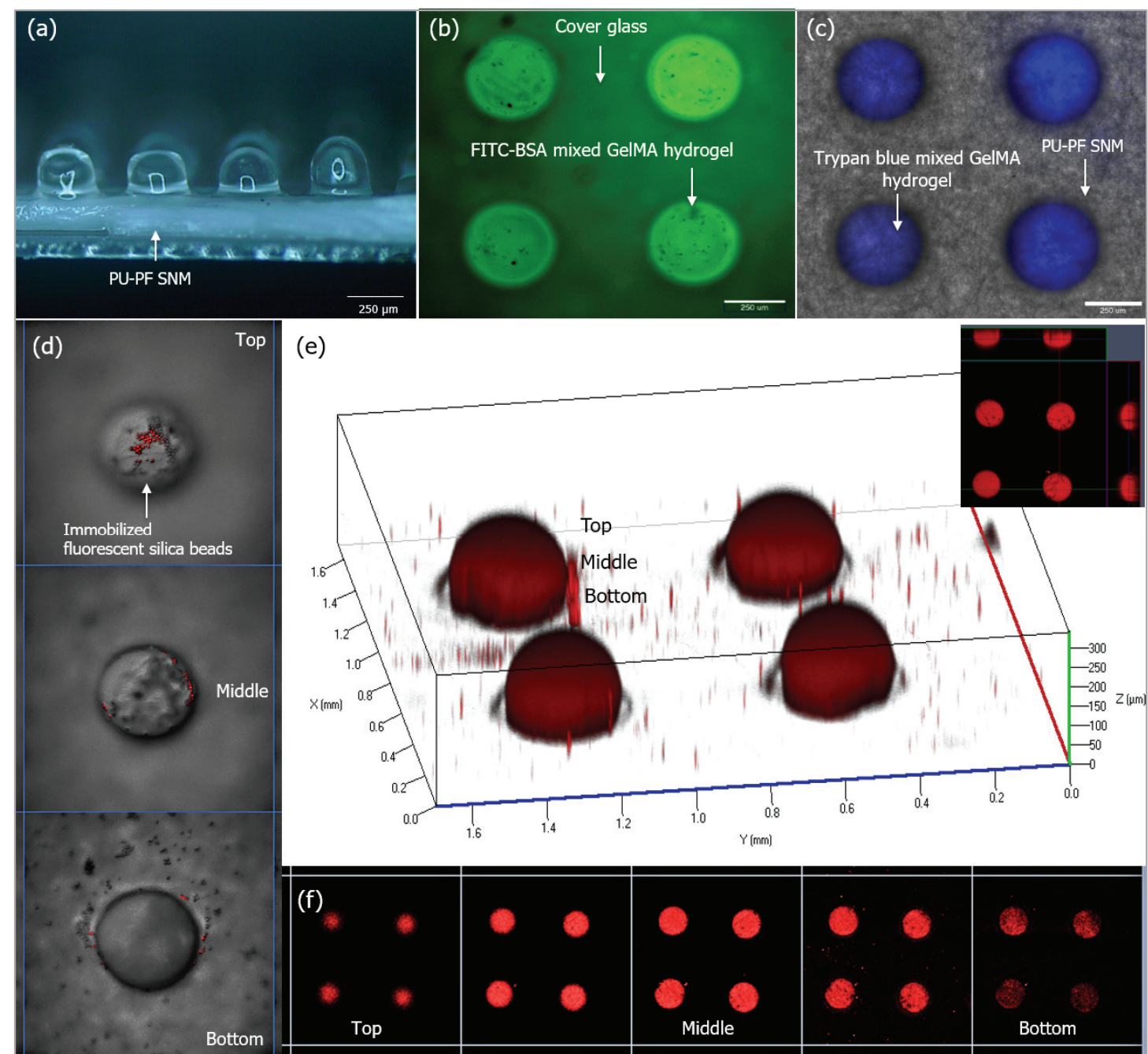

Figure 3. Micropatterned GelMA hydrogel. (a) Optical image of SNM, (b) FITC-BSA mixed fluorescence image on cover-glass, (c) Trypan-blue mixed GelMA micropattern on SNM, (d) fluorescent micro-particles immobilized on GelMA micropattern on cover-glass, (e) confocal microscopy Z-stack of rhodamine B mixed micropatterned GelMA hydrogel, and (f) image of gallery view of rhodamine B mixed micropatterned GelMA hydrogel on cover-glass.

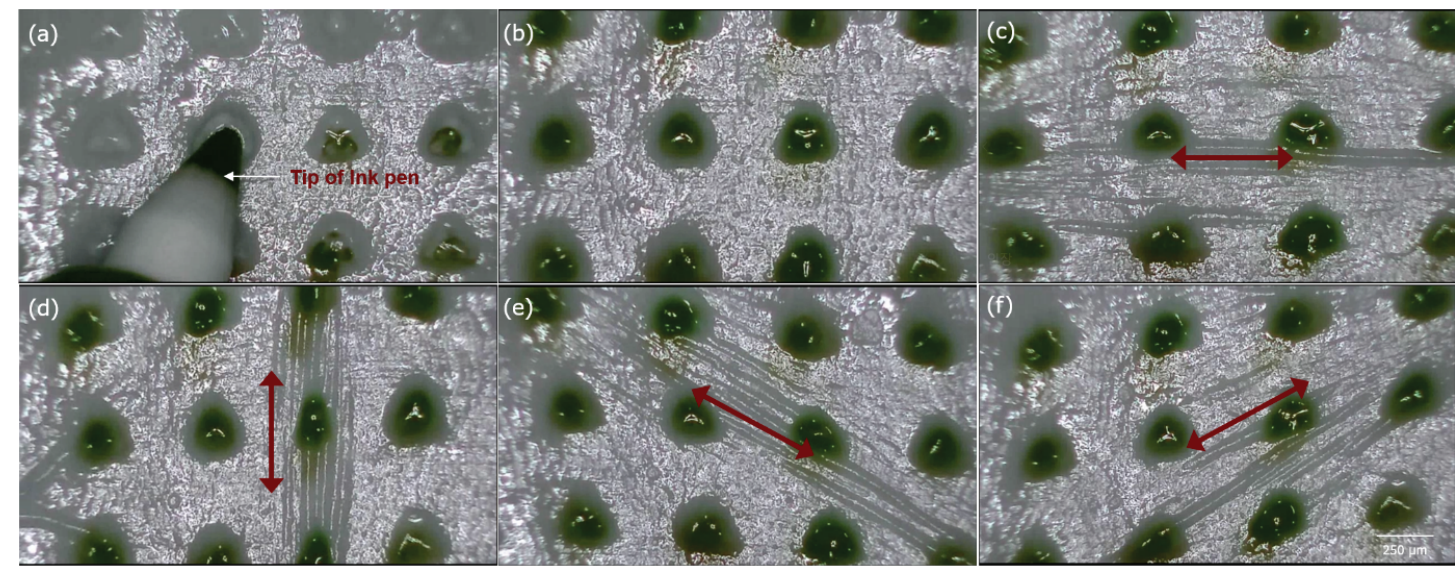

Figure 4. Compressive strength and adhesion tests. (a-b) Compressed GelMA hydrogel micropatterns on SNM, (c-d) horizontal and vertical stretching test, and (e-f) diagonally stretched SNM containing GelMA hydrogel micropatterns. 
Table 1. Relationship between the GelMA hydrogel, BMP-2, and SNM.

\begin{tabular}{|c|c|c|c|c|c|c|}
\hline \multicolumn{7}{|c|}{ Concentration of GelMA Hydrogel } \\
\hline & & $2.5 \%(w / v)$ & $5 \%(w / v)$ & $10 \%(w / v)$ & & \\
\hline \multirow{6}{*}{$\begin{array}{l}\text { Concentration } \\
\text { of } \\
\text { BMP-2 }(\mathrm{ng} / \mathrm{mL})\end{array}$} & \multirow{2}{*}{2000} & $\begin{array}{l}\text { Partial adhesion } \\
\text { Best cross-linking }\end{array}$ & $\begin{array}{l}\text { Partial adhesion } \\
\text { Good cross-linking }\end{array}$ & $\begin{array}{l}\text { Partial adhesion } \\
\text { Poor cross-linking }\end{array}$ & $\begin{array}{l}\text { PU } \\
\text { (hydrophobic) }\end{array}$ & \multirow{6}{*}{ - SNM } \\
\hline & & $\begin{array}{l}\text { Perfect adhesion } \\
\text { Best cross-linking }\end{array}$ & $\begin{array}{l}\text { Good adhesion } \\
\text { Best cross-linking }\end{array}$ & $\begin{array}{l}\text { Partial adhesion } \\
\text { Poor cross-linking }\end{array}$ & $\begin{array}{l}\text { PU-PF } \\
\text { (hydrophilic) }\end{array}$ & \\
\hline & 5000 & $\begin{array}{l}\text { No adhesion } \\
\text { Partial cross-linking }\end{array}$ & $\begin{array}{l}\text { Partial adhesion } \\
\text { Partial cross-linking }\end{array}$ & $\begin{array}{l}\text { No adhesion } \\
\text { Good cross-linking }\end{array}$ & $\begin{array}{l}\text { PU } \\
\text { (hydrophobic) }\end{array}$ & \\
\hline & 5000 & $\begin{array}{l}\text { Good adhesion } \\
\text { Partial cross-linking }\end{array}$ & $\begin{array}{l}\text { Partial adhesion } \\
\text { Good cross-linking }\end{array}$ & $\begin{array}{l}\text { Partial adhesion } \\
\text { Good cross-linking }\end{array}$ & $\begin{array}{l}\text { PU-PF } \\
\text { (hydrophilic) }\end{array}$ & \\
\hline & \multirow{2}{*}{10000} & $\begin{array}{l}\text { No adhesion } \\
\text { Partial cross-linking }\end{array}$ & $\begin{array}{l}\text { No adhesion } \\
\text { Partial cross-linking }\end{array}$ & $\begin{array}{l}\text { No adhesion } \\
\text { Partial cross-linking }\end{array}$ & $\begin{array}{l}\text { PU } \\
\text { (hydrophobic) }\end{array}$ & \\
\hline & & $\begin{array}{l}\text { No adhesion } \\
\text { Partial cross-linking }\end{array}$ & $\begin{array}{l}\text { Partial adhesion } \\
\text { Partial cross-linking }\end{array}$ & $\begin{array}{l}\text { Partial adhesion } \\
\text { Partial cross-linking }\end{array}$ & $\begin{array}{l}\text { PU-PF } \\
\text { (hydrophilic) }\end{array}$ & \\
\hline
\end{tabular}

uniform throughout the pattern; however, at the highest concentration of the GelMA hydrogel, FITC-BSA was observed locally, as shown in Figure S4 (Supporting Information). GelMA patterning containing BMP-2 on the surface of SNM requires hydrophilic rather than hydrophobic SNM, and it helps to penetrate the GelMA hydrogel rapidly and easily between SNM interlayers due to high surface tension. In addition, SNM as a substrate must possess sufficient transmittance to allow UV light to cross the GelMA in the PDMS mold through the SNM. As summarized in Table 1, change in BMP-2 concentration affected the pattern completeness of the GelMA hydrogel. Thus, the lowest concentration $(2.5 \% \mathrm{w} / \mathrm{v})$ of the GelMA hydrogel was used as the optimal condition for the best micropattern with $2000 \mathrm{ng} / \mathrm{mL}$ BMP-2.

\section{Analysis of Localized and Controlled Release}

Figure 5 shows the computationally simulated timedependent drug concentration distribution, which depended on the drug diffusion coefficients in the GelMA hydrogel models, for 3 days (i.e. 4,320 min). This simulation analyzes how the diffusion-driven release of BMP-2 might occur from the micropatterned GelMA hydrogel models of different concentrations. As expected, BMP-2 diffuses from the GelMA hydrogel into the surrounding, including the culture medium, over time, and concentration distribution is affected by the diffusion coefficients. We simulated three different diffusion coefficient models and investigated the spatiotemporal variations of BMP-2 for the entire domain, including the GelMA hydrogel structure and the surrounding after every $60 \mathrm{~min}$. At 60 min, BMP-2 molecules diffused slightly through the interface between the GelMA hydrogel and the surrounding, although the diffusion rates for each case appeared to differ slightly. At 1,440 min (1 day), the highest density model retained more BMP-2 (approximately $0.6 \mathrm{~mol} / \mathrm{m}^{3}$ ) at the center of the patterned GelMA hydrogel, whereas other lower density models retained approximately $0.5 \mathrm{~mol} / \mathrm{m}^{3}$ and $0.3 \mathrm{~mol} /$ $\mathrm{m}^{3}$. In the surroundings in the vicinity of the GelMA hydrogel boundary, the spatial distribution of BMP-2 for the lowest density model $(2.5 \% \mathrm{w} / \mathrm{v})$ shown in Figure $5 \mathrm{a}$ was distinct from that in the other higher density models shown in Figure 5c,e. More BMP-2 diffuse to the region lacking BMP-2 in the low density model than in the other higher density models. Furthermore, owing to the possible diffusion effects from the neighboring GelMA hydrogel micropatterns on both sides, fluxes through either side boundary were not observed. The distribution showed a convex-roof shape due to an upward movement along the side boundary. At 2,880 min (2 days), the BMP-2 distribution appeared to be apparently flat in the surroundings near the GelMA hydrogel pattern for the lowest density model. The highest density model $(10 \%$ $\mathrm{w} / \mathrm{v}$, shown in Figure 5e) still maintained a semicircular shape of BMP-2 distribution, as high density of GelMA reduces diffusion of BMP-2 out of the patterned GelMA hydrogel into the surroundings. The middle density model (5\% w/v, Figure $5 \mathrm{c})$ also showed a gradually flattening BMP-2 distribution in the surrounding. On day 1 , the concentrations at the center of each model decreased to half of the initial value. At 4,320 $\mathrm{min}$ (3 days), the highest density model finally showed a convex-roof shaped BMP-2 distribution curve in the surrounding, whereas almost flat BMP 


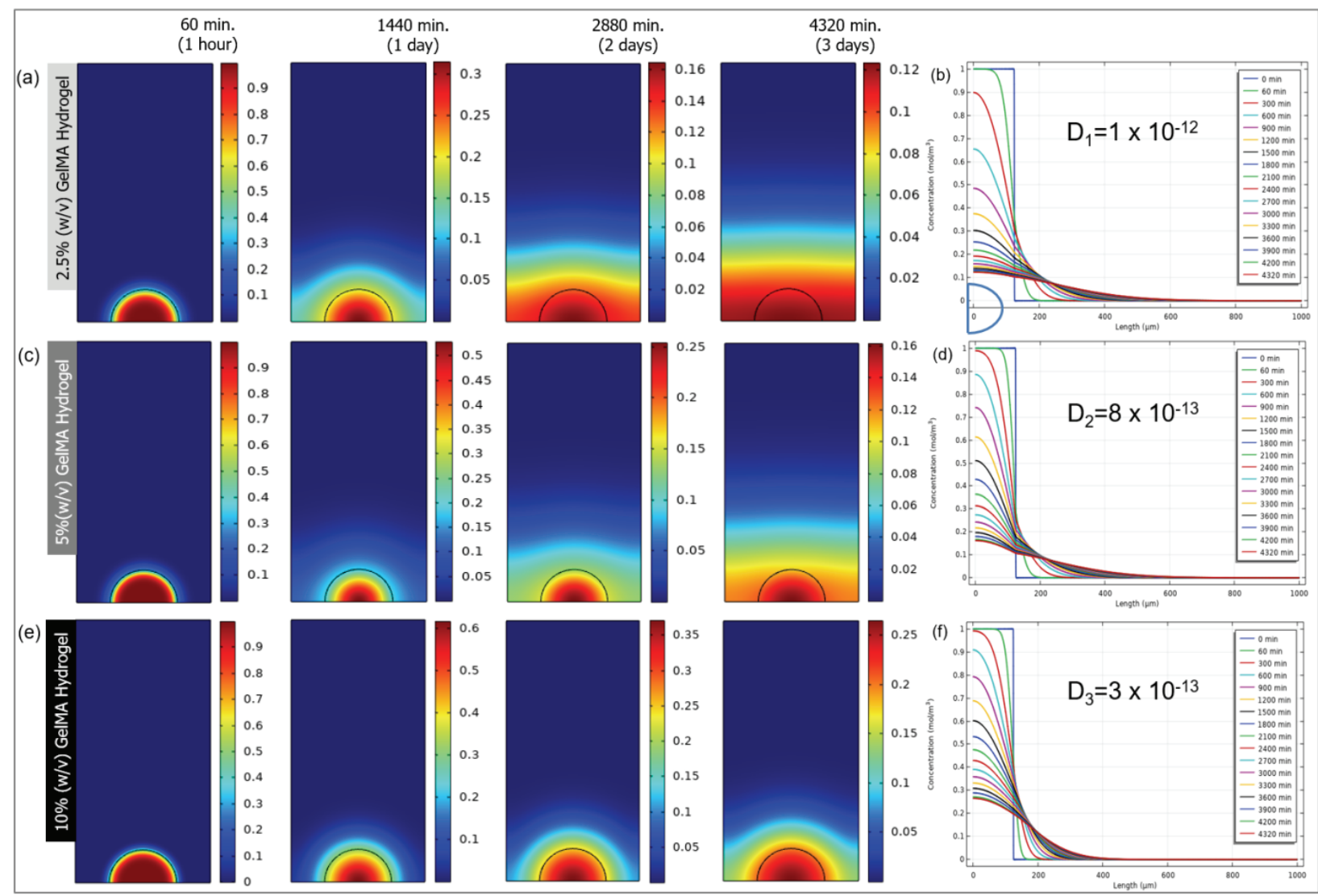

Figure 5. Computational simulation model based on the Millington and Quirk diffusivity model. (a-b) Lowest (2.5\% w/v), (c-d) middle $(5 \% \mathrm{w} / \mathrm{v})$, and $(\mathrm{e}-\mathrm{f})$ highest $(10 \% \mathrm{w} / \mathrm{v})$ concentration of GelMA hydrogel.

-2 distribution in the surroundings was observed for the other lower density models; this configuration is expected to be retained with gradual changes in concentration, to the end of boundary of the analysis domain over time. Interestingly, the concentrations of the highest density model were almost 2-fold higher than those of the lowest density model for 1,2 , and 3 days. As the BMP-2 release from the GelMA modeled as a 2D semicircle, the concentration of the drug in the GelMA gradually decreased over time due to diffusion, and the decrease rates differed with the densities of the GelMA hydrogel. Eventually, the BMP2 concentration in the GelMA hydrogel approached that of the surrounding from the initial value. Figure $5 b$,d,f shows the spatiotemporal variations of BMP-2 concentrations along a hypothetical cut line connecting the centers of both the patterned GelMA hydrogel and the top boundary of the surrounding, as indicated in Figure 5a,c,e. The initial concentration distribution shows a vertical drop at $125 \mu \mathrm{m}$ corresponding to the pattern radius, indicating that BMP-2 molecules were uniformly distributed over the patterned volume. The diffusion occurred gradually into the surrounding. In the early stages, the distribution showed a steep gradient near the interface in both domains. As time progressed, the molecules permeated the surrounding and the gradient changed gradually. As expected, the highest concentration model $(10 \% \mathrm{w} / \mathrm{v})$ showed the slowest gradient variation in concentration than the other lower density $(2.5$ and $5 \% \mathrm{w} / \mathrm{v})$ models. In addition, at the last stage, the concentration at the center for the highest density model was the highest, indicating that the extent of BMP-2 retention in the GelMA hydrogel was the strongest. To determine whether the GelMA hydrogel micropattern can improve the sustainability of the BMP-2 drug carrier and to predict the release profile of BMP-2 from the GelMA hydrogel, FITCBSA, instead of BMP-2 (similar amounts), was loaded on the hydrogel. Using fluorescent microscopy, the amount of FITC-BSA (excitation: $485 \mathrm{~nm} / \mathrm{emission}$ : $535 \mathrm{~nm}$ ) released from the micropatterns to the outer phosphate buffered saline (PBS) due to difference in diffusion was measured over time and the emission amount was calculated for 6 days (Figure 6). Initially, 


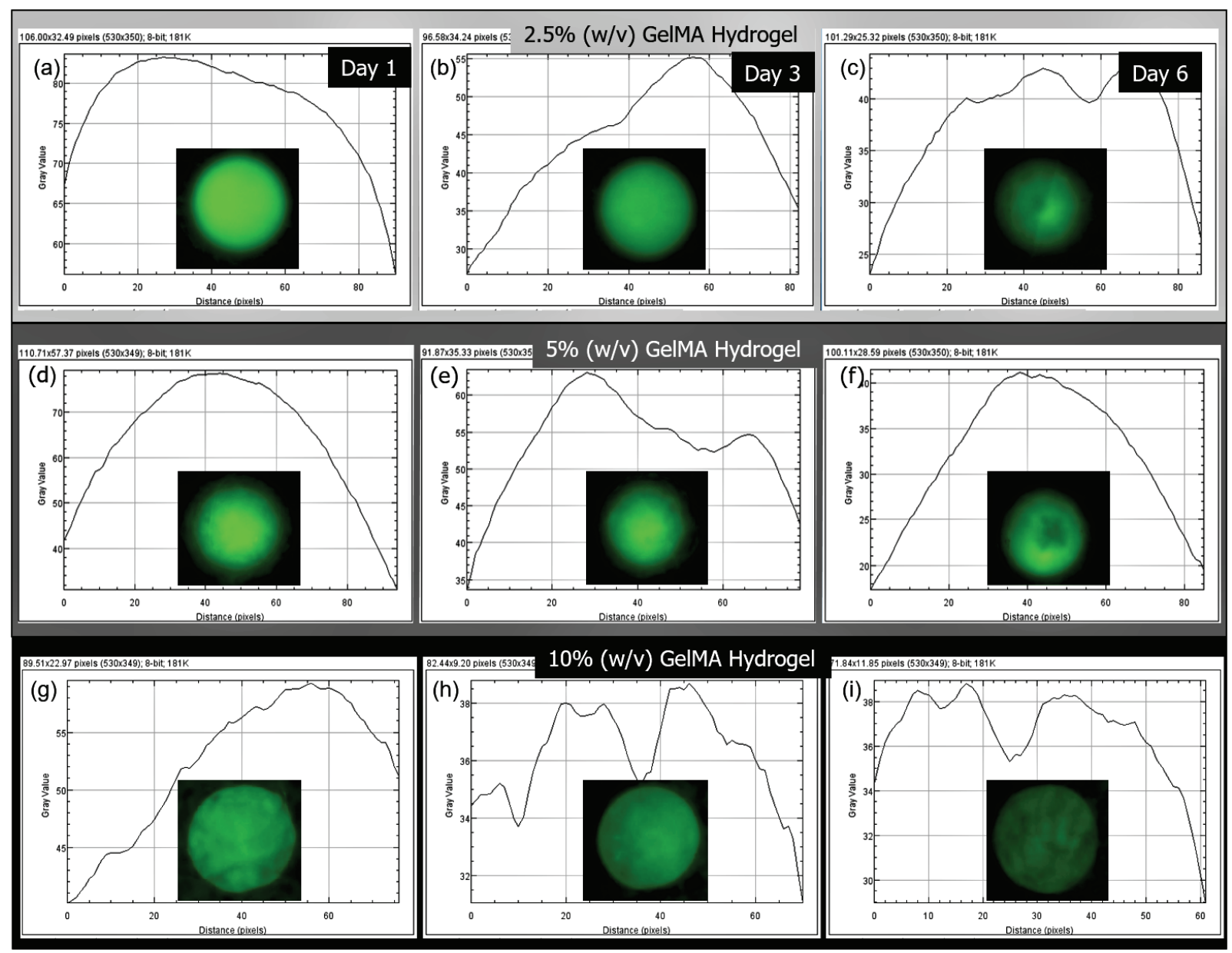

Figure 6. Measurement of change in fluorescence intensity of FITC-BSA in patterned GelMA hydrogel for 6 days. (a-c) Lowest $(2.5 \% \mathrm{w} / \mathrm{v}),(\mathrm{d}-\mathrm{f})$ middle $(5 \% \mathrm{w} / \mathrm{v}),(\mathrm{g}-\mathrm{i})$ highest $(10 \% \mathrm{w} / \mathrm{v})$ concentration of GelMA hydrogel.

each fixed FITC-BSA intensity was normalized to 100. As shown in Figure 6a,d, and g on day 1, the low concentration case showed a rapid decrease of approximately $75 \%$ fluorescence intensity $(25.1 \mathrm{a}$. u.) due to the convulsive diffusion at the initial stage, and kept the decreased intensity for 6 days (22.0 a. u. on day 3 and 21.7 a. u. on day 6). Even at the medium and high concentrations, the rapid decreases of about $65 \%$ (35. 2 a. u.) and $52 \%$ (49.1 a. u.), respectively, were observed only on the first day. On the $3^{\text {rd }}$ day, the reduction rates of only $4 \%$ (34.3 a. u.) and $6 \%$ (45.2 a. u.) were observed, comparable to the first day (Figure $6 \mathrm{~b}, \mathrm{e}$, and $\mathrm{h}$ ), and only the smallest reduction rates of $5 \%$ (33.7 a. u.) and $7 \%$ (44.1 a. u.) were recorded on the last $6^{\text {th }}$ day, respectively (Figure $6 \mathrm{c}, \mathrm{f}$, and i). It has been known that when the hydrogel is cross-linked, the swelling of the hydrogel is maximized for 24 hours. As the concentration of the hydrogel decreases, the swelling degree increases and the density dec- reases, whereas the swelling degree decreases and the density increases when the concentration increases ${ }^{41}$. Thus, the swelling of the GelMA hydrogel by crosslinking results in the volume changes of the patterns and redistribution of density within the patterns. Under the same diffusion condition, the diffusive transfer of FITC-BSA at the low concentration of GelMA is rapidly and easily made toward PBS solution. At the medium and high concentrations, the initial diffusion rates are shown to be lowered by about $10 \%$ and $23 \%$, respectively, due to the relatively high density of the patterns. Although the experiments lasted for 6 days, the results were consistent with those of the typical and ideal drug release profiles, which shows burst release in low density carriers and sustained release in high density carriers ${ }^{42-45}$. These results are similar to those obtained using computational simulations where BMP-2 is encapsulated in different concentrations of the GelMA hydrogel, indicating that 

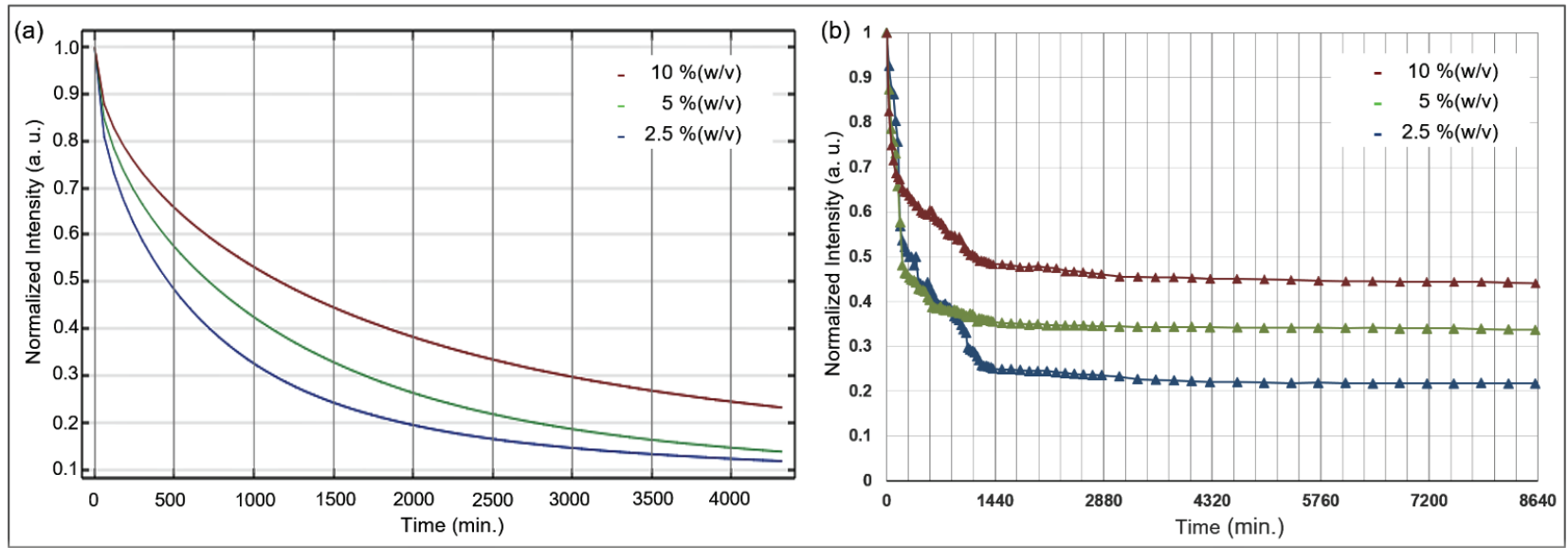

Figure 7. Normalized release profiles of the FITC-BSA from the lowest, middle, and highest GelMA hydrogel. (a) Computational simulation for 3 days and (b) FITC-BSA loaded GelMA hydrogel micropattern after 6 days.

BMP-2 can be released at different rates via localized and controlled release within the GelMA hydrogel micropatterns. Finally, we compared the experimental results with those of the simulation study. Although the simulation study lasted for only 3 days (Figure 7a) and the experiments were conducted for 6 days (Figure $7 \mathrm{~b}$ ), the results of the simulation study and those obtained from the first 3 days of the experiments were significantly similar. This indicated that the experiments were performed under optimal conditions for reliable immobilization and release of BMP-2 from the patterned GelMA hydrogel. Based on the FITC-BSA release profiles in terms of the GelMA concentrations resulted from both simulation and experiments, the local release of BMP-2 would be expected to be controllable for more conditions.

\section{Osteogenic Differentiation of MG-63 Cells after BMP-2 Release}

To investigate the relationship between osteogenic differentiation and BMP-2 release, MG-63 cells were cultured in the absence or presence of BMP- 2 on $2.5 \%$ (w/v) GelMA hydrogel micropattern and analyzed using actin filament staining and Alizarin Red S (ARS) staining (Figure 8). Although the SNM used as a substrate has an extracellular matrix-like surface structure, which can provide a good environment for cell attachment and proliferation ${ }^{44,46,47}$, MG-63 cells were observed to adhere primarily around the GelMA pattern. In particular, when BMP-2 was immobilized, the cells were observed to grow mainly when they were attached to the pattern and were in the vicinity of the pattern. When the cytoskeleton of the cells was observed using actin filament staining, we observed that a relatively rich and dense actin network was formed between MG-63 cells in the presence of BMP-2. In addition, in the absence of BMP-2 in the GelMA pattern, the cells tended to be located around the pattern in random direction (Figure 8a-c); however, in the case of BMP-2 immobilization, the cells were oriented toward the central axis of each pattern (Figure $8 \mathrm{~d}-\mathrm{f}$ ). In terms of osteogenic differentiation, ARS staining was used to colorimetrically distinguish the extent of calcium deposition as a result of osteoblastic differentiation. Generally, the amount of calcium in the medium increases when MG-63 cells induce bone differentiation, and the calcium ion forms a strong covalent bond with oxygen or hydroxyl group in ARS, resulting in an orange-red precipitate 48. Six days after seeding the MG-63 cells, calcium deposited nodules were observed under both conditions. However, as shown in Figure 9, the calcium deposits formed by MG-63 cells on the surface of GelMA were significantly higher in the presence of BMP-2 (Figure 9c,d) than in the control group (Figure 9a,b). For quantitative analysis, the area of calcium deposition was calculated using the Image J software. In the BMP-2 group, the average deposition area was approximately $32 \mathrm{~mm}^{2}$, whereas it was approximately $86 \mathrm{~mm}^{2}$ in the control group. These results show that BMP-2 was locally released from the GelMA micropattern, which significantly affected the osteogenic differentiation of MG-63 cells. The calcium deposition correlated with the changes in the cytoskeleton after the release of BMP-2 (Figure 8). Despite these meaningful results, the clinical application of the BMP-2 carrier is limited due to the short duration of 6 days and the in-vitro condition based on cell culture medium. Therefore, further studies such 


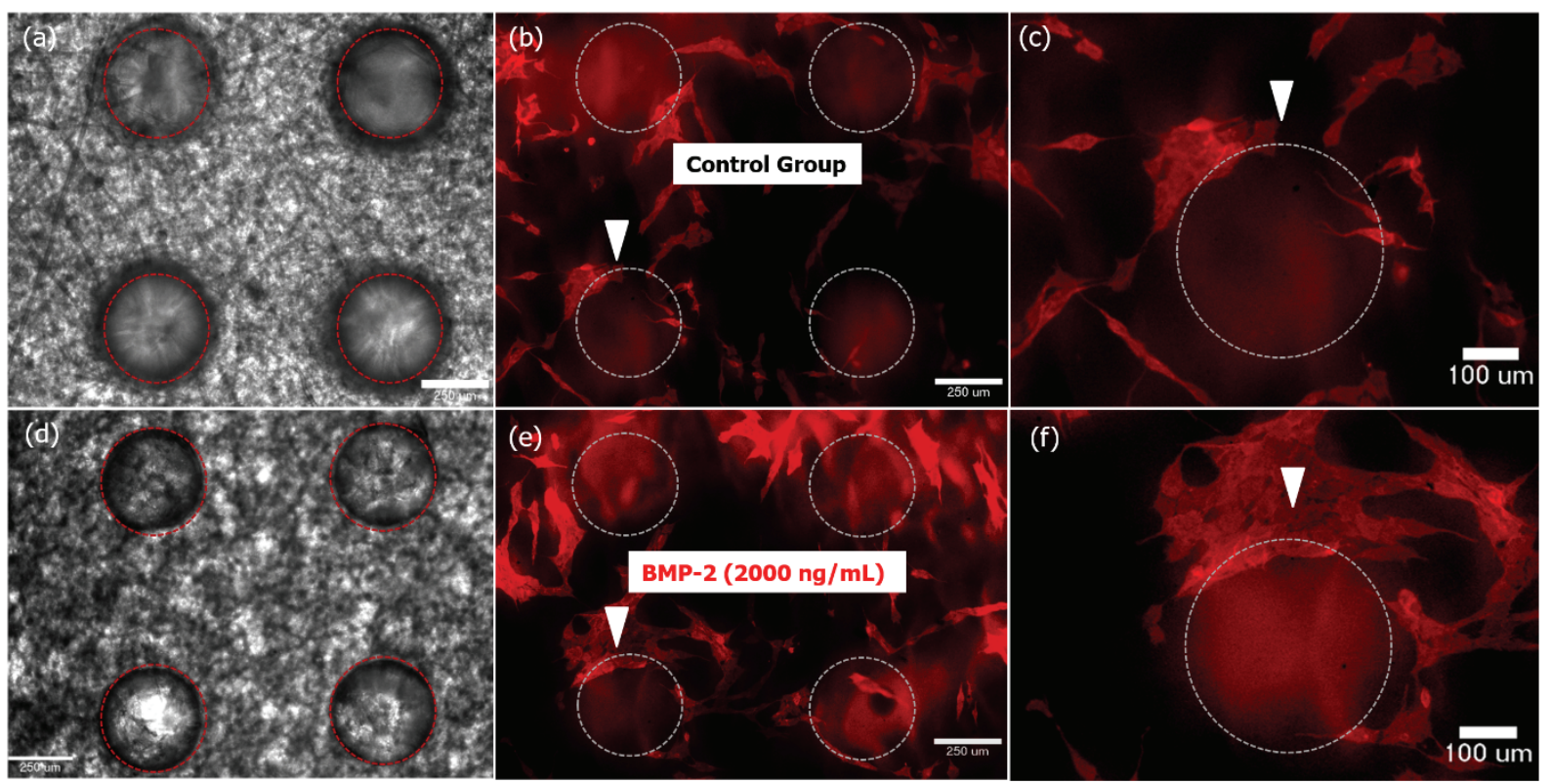

Figure 8. Actin filament staining of MG-63 cells after 6 days. (a-c) In the absence of BMP-2 and (d-f) in the presence of 2000 $\mathrm{ng} / \mathrm{mL}$ BMP-2 released from the $2.5 \%$ (w/v) GelMA hydrogel micropattern on SNM.

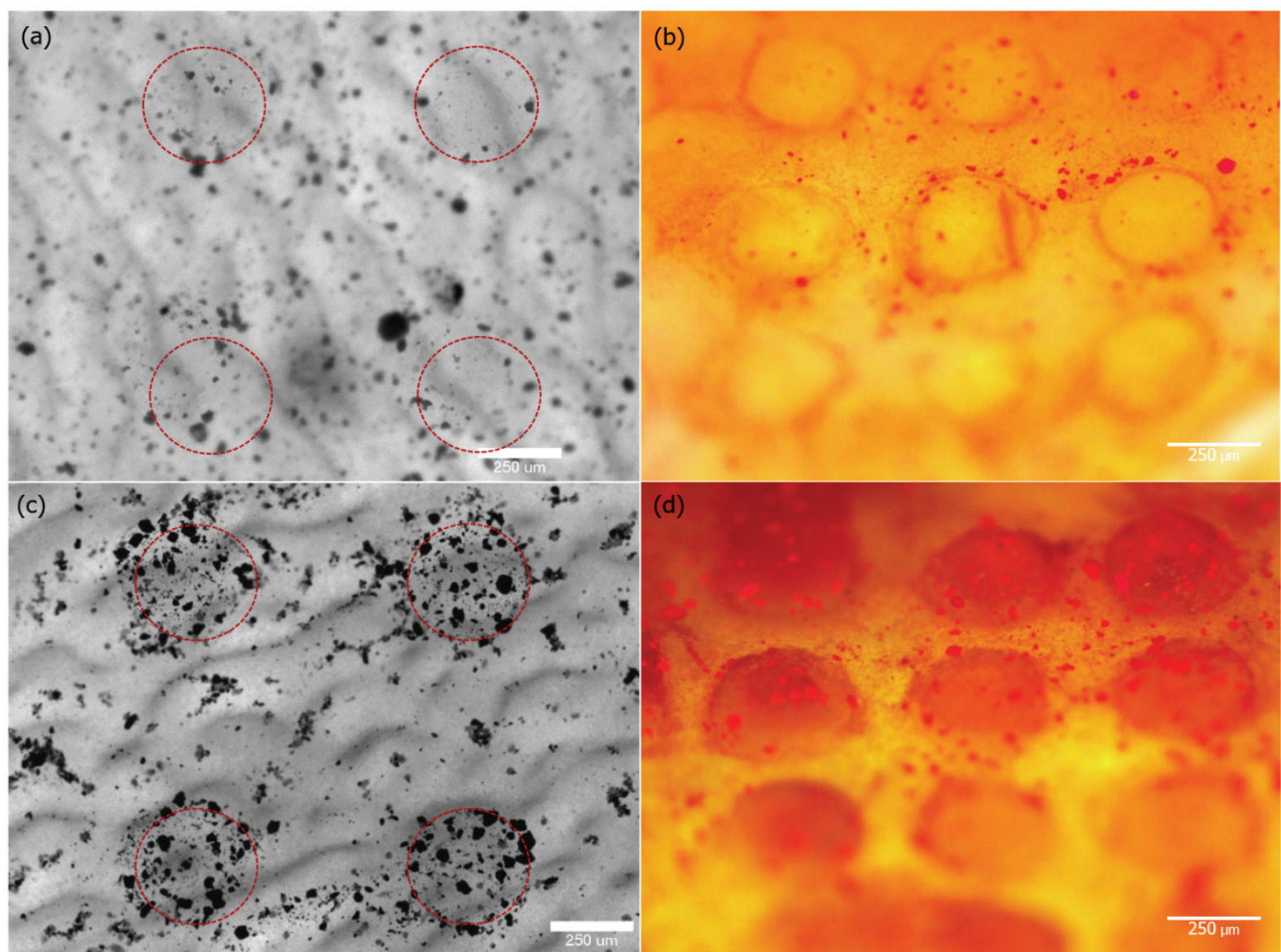

Figure 9. Osteogenic differentiation of MG-63 cells with ARS staining after 6 days. (a-b) In the absence of BMP-2 and (c-d) in the presence of $2000 \mathrm{ng} / \mathrm{mL}$ BMP-2 released from the $2.5 \%(\mathrm{w} / \mathrm{v})$ GelMA hydrogel micropattern on SNM. 
as in vivo testing and alkaline phosphatase activity (ALP) assay are still needed.

\section{Conclusion}

In the clinical procedure of bone grafting on a defective site, a polymer-based barrier membrane is used to prevent invasion of connective tissue into the bone regeneration area $^{49}$. However, although growth factors are used to promote bone formation, their use has been limited owing to problems such as burst release and action at the ectopic site depending on the growth factor carrier ${ }^{50}$. By optimizing the concentrations of BMP-2 and GelMA hydrogels for immobilization and micropatterning, respectively, we demonstrate that BMP-2 is easily immobilized into micropatterned GelMA hydrogels and that the local release of BMP -2 is effectively controlled. The GelMA hydrogels provide an environment in which BMP-2 can be homogeneously laden, while the SNM supported transmission of UV light, allowing the GelMA hydrogels containing BMP-2 to crosslink rapidly on the SNM surface. The behavior of MG-63 cells in bone differentiation medium depended on the distribution of BMP-2, indicating that bone differentiation of MG63 cells can be manipulated by controlling the immobilized BMP-2 in the GelMA hydrogel micropattern. The proposed method can be applied to various clinical treatments requiring controlled delivery of growth factors and cells, such as in bone regeneration, where the pattern size and concentration of BMP-2 can be changed depending on the size of the bone defect or the type of bone graft.

\section{Materials and Methods}

\section{PDMS Concave Micromold for Micropatterning}

A polymeric concave micromold was used to facilitate BMP-2 immobilization into the GelMA hydrogel. For fabricating a PDMS (Dow Corning, MI, USA)based concave micromold using a replication process, a well-defined photo-lithography method was used with the photosensitive epoxy resin (SU-8, MicroChem, MA, USA $)^{51,52}$. A hemispheric SU-8 polymeric convex master mold, which commonly uses an epoxy-based negative photoresist according to a published protocol, was used ${ }^{51}$. Briefly, to make a master mold, a perforated SU-8 shadow mask layer with arrayed holes (diameter: $250 \mu \mathrm{m}$ ) was designed. Then, the perforated SU-8 shadow layer was exposed to UV light for SU-8 polymerization with the designed photomask film. Following curing and development of the SU-8 photoresist, the Kapton film and SU-8 were separated from a Si-wafer, and the Kapton film was carefully removed from the SU-8 photoresist layer, which was used as a shadow mask. The radius of each pattern was $250 \mu \mathrm{m}$, and they appeared at intervals of $250 \mu \mathrm{m}$. Finally, to replicate the concave micromold, the PDMS solution was mixed at a 10 (base):1.2 (curing agent) weight ratio. The mixed solution was stirred for $10 \mathrm{~min}$ with a stirring stick and vacuumed to eliminate residual bubbles. The PDMS solution was poured into the prepared SU-8 master mold, and heated to $80{ }^{\circ} \mathrm{C}$ for $30 \mathrm{~min}$. Then, after annealing to the hotplate, the replicated PDMS concave micromold was separated from the SU-8 master mold.

\section{GelMA Hydrogel Micropatterns on SNM}

Localized GelMA hydrogel patterning was performed via in situ photo-polymerization using prepared the PDMS concave micromold and electrospun SNM. After autoclaving (for sterilization), the surfaces of the PDMS concave micromold and SNM were treated with $60 \mathrm{~W}$ oxygen plasma (Cute, Femto-Science, Inc.) for easy filling and adsorption of GelMA solution (Figure $1 \mathrm{~b}, \mathrm{c})^{53}$. Then, $1 \mathrm{~mL}$ of $2.5,5$, and $10 \%$ (w/v) GelMA solution was filled onto the PDMS mold and the excess solution was removed using a rubber scraper. Next, SNM $(15 \times 15 \mathrm{~mm})$ was used to cover the PDMS concave micromold. For photo-crosslinking the SNM, the covered SNM surface was exposed to UV light (Omnicure S2000, Maritimes, Canada) (wavelength: $360 \mathrm{~nm}$, intensity: $10,000 \mathrm{~mW} / \mathrm{cm}^{2}$ ) at distance of $8 \mathrm{~cm}$ for $30 \mathrm{~s}^{54}$. The photo-crosslinked GelMA hydrogel micropatterns were obtained by peeling the SNM from the PDMS mold as shown in Figure 2b,c. As shown in Figure $3 \mathrm{~b}, \mathrm{c}$, to observe and visualize the photo-cross-linked GelMA hydrogel micropatterns on SNM, 2.5\% (w/v) GelMA solution was mixed with trypan blue (Sigma-Aldrich, St, Louis, MO, USA) and FITC-BSA $(70 \mathrm{kDa})$ (Sigma-Aldrich) solution. In addition, to determine the BMP-2 immobilization ability of various materials, $5 \mu \mathrm{m}$ fluorescent silica beads were mixed and cross-linked with the GelMA hydrogel and scanned using confocal microscopy. The images were analyzed to determine whether the beads were homogeneously dispersed in the hemispherical micropatterned GelMA hydrogel (Figure 3d-f). 


\section{Mechanical Properties and Adhesion Stability}

A digital force gauge meter (FGV-50XY, Shimpo Co. Japan) was used for the measurement of the compressive strength of the micropatterned GelMA hydrogel on the SNM. Deformation was observed by pressing the micropatterned GelMA hydrogel with the tip of an ink pen (Movie S1, Supporting Information). Simultaneously, for evaluating the adhesion between the GelMA hydrogel micropatterns and the SNM surface, the GelMA hydrogel micropatterned SNM was stretched several times in the horizontal, vertical, and diagonal directions and the separation of the GelMA micropattern from the SNM was observed (Movie S2, Supporting Information). In addition, the tensile strength was examined using a micro-universal testing machine (UTM) (LR10K-plus, Lloyd Instruments Ltd., UK) under a crosshead speed of 50 $\mathrm{mm} / \mathrm{min}$ at room temperature. The specimens were prepared as rectangles $(10 \times 50 \mathrm{~mm})$ containing GelMA hydrogel micropatterns. During the testing, a moisturizing mist was intermittently sprayed onto the specimens to prevent dehydration of the GelMA hydrogel (Figure S5, Supporting Information).

\section{Estimation of the Release Profile of BMP-2 from the Micropatterned GelMA Hydrogel}

To assess the fundamental release profile determining the concentration of the GelMA hydrogel, the Millington and Quirk model for drug transport was theoretically studied using a computational porous material with effective transport properties. The surroundings were modeled in the shape of a rectangle of 500 $\mu \mathrm{m}$ width and $1000 \mu \mathrm{m}$ height, while the actual depth of the culture medium in the experiments was several orders of magnitude higher than the height of the surroundings of the simulation. Therefore, the top boundary condition of the surroundings was defined to be of the external convection type of flux with a mass transfer coefficient. The boundary condition of both sides was set to no flux of drugs through the boundary via diffusion from the neighboring surroundings. The bottom surface of the surrounding was also under no flux condition. The half-circular boundary of the surroundings adjacent to the GelMA top surface was assumed to have the same concentration as that of the surface. A simple 2D model analyzed using the COMSOL ${ }^{\circledR}$ Multiphysics software, a commercial finite element method (FEM)-based simulation tool (version 5.4, module Chemical Species Transport and Transport of Diluted Species in Porous Media, COMSOL Inc., Burlington, MA, USA), was used for numerical analysis to emulate the diffusive transfer of BMP-2 in GelMA hydrogel under three different concentrations. As the actual size of the pores in hydrogel materials is typically several orders of magnitude smaller than the model domain to be analyzed and is difficult to be described in detail, a simple 2D porous model with effective porosity was defined using the simulation. The equation for the time-dependent model is based on the Millington and Quirk diffusivity model as follows:

$$
\begin{aligned}
& \frac{\partial \mathrm{c}}{\partial \mathrm{t}}+\nabla \cdot\left[-\left(\mathrm{D}_{\mathrm{D}}+\mathrm{D}_{\mathrm{e}}\right)\right] \nabla \mathrm{c}=0 \\
& D_{e}=\frac{\epsilon_{p}}{\tau_{F}} D_{F}
\end{aligned}
$$

where $c$ denotes the concentration $(\% \mathrm{w} / \mathrm{v}), D_{D}$ the dispersion coefficient $\left(\mathrm{m}^{2} / \mathrm{s}\right), D_{e}$ the diffusion coefficient $\left(\mathrm{m}^{2} / \mathrm{s}\right), \in_{P}$ the porosity, $\tau_{F}$ the effective diffusivity which is equal to $\epsilon_{P}^{-1 / 3}$, and $D_{F}$ the fluid diffusion coefficient $\left(\mathrm{m}^{2} / \mathrm{s}\right)$. This computational simulation solves only diffusion and dispersion problem by mass transfer in porous media due to concentration gradient, and no forced convection mechanism was considered in the GelMA hydrogel domain. The initial condition of drug concentration was set to $c_{\text {sur }}\left(t_{0}\right)=0$. As shown in Figure $4 \mathrm{a}-\mathrm{c}$, both GelMA and the surroundings were discretized in 2D-triangular meshes using predefined extra fine element size with maximum diameter of $20 \mu \mathrm{m}$. For high accuracy and rapid convergence of the results, the half-circular boundary between patterned GelMA hydrogel and the surroundings were also discretized using the boundary layer properties, with 8 layers for both sides and boundary layer stretching factor of 1.2. The maximum element size was $5 \mu \mathrm{m}$. The structure of GelMA hydrogel micropattern model for the simulation is assumed to be a half circle with a radius of $125 \mu \mathrm{m}$, and the space between the neighboring structures is $250 \mu \mathrm{m}$, which is based on the actual patterned GelMA hydrogel dimensions. Instead of simulating the full-scale actual micropattern ( 8 by 8 ), only one structure and the surroundings in the vicinity were analyzed. The initial condition of drug concentration in the GelMA hydrogel domain is defined as a constant, $c\left(t_{0}\right)=c_{0}$. The boundary condition at the bottom surface of the GelMA hydrogel was set to no flux of drug through the boundary, while the external convection flux was considered the boundary condition at the top surface, which was not due to forced convective flux, but due to diffusion arising from the concentration differences between the micropattern 
and the surroundings as described in the following equation.

$$
-\left(D_{D}+D_{e}\right) \nabla c \cdot \vec{n}=k_{m}\left(c-c_{\text {sur }}\right)
$$

Where $\vec{n}$ denotes the normal vector, $k_{m}$ is the mass transfer coefficient $(\mathrm{m} / \mathrm{s})$ in GelMA, and $c_{\text {sur }}$ denotes the concentration $[\%(\mathrm{~m} / \mathrm{v})]$ in the surrounding domain. In addition, to predict the release profile of BMP-2 (30 kDa) (which will be immobilized later), the release profile of FITC-BSA $(70 \mathrm{kDa})$ from the micropatterned GelMA hydrogel was assessed using an inverted fluorescence microscope (Axiovert 200M, Carl Zeiss, Darmstadt, Germany). The samples were prepared with the lowest, middle, and highest concentrations of GelMA solution $(2.5,5$, and $10 \% \mathrm{w} / \mathrm{v})$ (Figure 5). To emulate the in vivo condition, $3 \mathrm{~mL}$ PBS solution was changed each time after sampling over 6 days. The same spot of each sample was monitored daily to observe the changes in the fluorescence intensity of FITC-BSA measured over time.

\section{Evaluation of Osteogenic Differentiation and Morphology}

To evaluate the effect of osteogenic differentiation, including morphology, on the localized and controlled release of BMP-2, human osteoblast-like MG-63 cells (American Type Culture Collection, Rockville, MD, USA) were cultured onto the GelMA hydrogel micropatterned SNM for 6 days. In particular, before the pattering process, the SNM was fixed onto the bottom of the cell culture dish using half-cured PDMS solution and dried for preventing the floating of SNM in the culture media. The cell density was $1 \times 10^{6}$ cells/ $\mathrm{mL}$, and $100 \mu \mathrm{L}$ cells were seeded onto the BMP-2 (2000 ng/mL)-immobilized GelMA hydrogel micropatterned SNM. In addition, MG-63 cells were simultaneously cultured on SNM without BMP-2 as the control group. The cells were cultured in cell media $[10 \%(\mathrm{w} / \mathrm{w})$ Dulbecco's modified Eagle's medium (DMEM)] (Gibco, CA, USA) supplemented with 1\% $(\mathrm{w} / \mathrm{w})$ fetal bovine serum (FBS) (Gibco) and 1\% (w/w) penicillin/streptomycin (Gibco) in a humidified atmosphere containing $5 \% \mathrm{CO}_{2}$ at $37^{\circ} \mathrm{C}$. After 6 days, the actin filaments were stained with Alexa Fluor 568 phalloidin fluorescent dye (Invitrogen, Eugene, OR, USA) to determine the cytoskeletal organization in MG-63 cells. Image-iTTM FX signal enhancer was used to enhance the signal-to-noise ratio of fluorescently labeled cells, and fluorescent images were acquired and processed using an Axiovert 200 inverted microscope. Osteogenic differentiation was evaluated by staining the calcium deposits using the ARS staining kit (pH 4.1-4.3; Sigma-Aldrich) ${ }^{55}$. First, the culture medium was removed, and the cells were gently washed thrice with PBS. Next, the cells were fixed with $4 \%(\mathrm{w} / \mathrm{v})$ formaldehyde for $15 \mathrm{~min}$ at room temperature. Then, the cells were washed with distilled water, followed by the addition of $2 \%(\mathrm{w} / \mathrm{v})$ solution of $60 \mathrm{mM}$ ARS, the $\mathrm{pH}$ to $4.1-4.3$ of which was adjusted using $\mathrm{NH}_{4} \mathrm{OH}$ and $\mathrm{HCl}$ solutions. After filtering the ARS solution with a $0.2 \mu \mathrm{m}$ filter, $1 \mathrm{~mL}$ of the ARS solution was dropped onto the SNMs and incubated for $1 \mathrm{~h}$ at $4{ }^{\circ} \mathrm{C}$. Then, the stained cells were photographed to analyze the calcium deposits induced by released BMP-2.

Acknowledgements This work is supported by a grant from the Basic Science Research Program through the National Research Foundation of Korea (NRF) funded by the Ministry of Education (2017 R1D1A1A0201935) and 2016 Research Grant form Kangwon National University (No. 520160439).

Conflict of Interests The authors declare no competing financial interests.

\section{References}

1. Yamamoto, M., Hokugo, A., Takahashi, Y., Nakano, T., Hiraoka, M. \& Tabata, Y. Combination of BMP2-releasing gelatin/beta-TCP sponges with autologous bone marrow for bone regeneration of X-rayirradiated rabbit ulnar defects. Biomaterials 56, 18-25 (2015).

2. Wang, C.K., Ho, M.L., Wang, G.J., Chang, J.K., Chen, C.H., Fu, Y.C. \& Fu, H.H. Controlled- release of rhBMP-2 carriers in the regeneration of osteonecrotic bone. Biomaterials 30, 4178-4186 (2009).

3. Santo, V.E., Duarte, A.R., Popa, E.G., Gomes, ME., Mano, J.F. \& Reis, R.L. Enhancement of osteogenic differentiation of human adipose derived stem cells by the controlled release of platelet lysates from hybrid scaffolds produced by supercritical fluid foaming. J. Controlled Release 162, 19-27 (2012).

4. Lee, S.S. , Hsu, E.L., Mendoza, M., Ghodasra, J., Nickoli, M.S., Ashtekar, A., Polavarapu, M., Babu, J., Riaz, R.M., Nicolas, J.D., Nelson, D., Hashmi, S. Z., Kaltz, S.R., Earhart, J.S., Merk, B.R., McKee, J. S., Bairstow, S. F., Shah, R. N., Hsu, W. K. \& Stupp, S.I. Gel scaffolds of BMP-2-binding peptide amphiphile nanofibers for spinal arthrodesis. $A d v$. Healthcare Mater. 4, 131-141 (2015). 
5. Yao, Q., Liu, Y., Selvaratnam, B., Koodali, R.T. \& Sun, H. Mesoporous silicate nanoparticles/3D nanofibrous scaffold-mediated dual-drug delivery for bone tissue engineering. J. Controlled Release 279, 69-78 (2018).

6. Tuncaboylu, D.C., Friess, F., Wischke, C. \& Lendlein, A. A multifunctional multimaterial system for on-demand protein release. J. Controlled Release 284, 240-247 (2018).

7. Oliveira, H.F.D., Weiner, A.A., Majumder, A. \& Shastri, V.P. Non-covalent surface engineering of an alloplastic polymeric bone graft material for controlled protein release. J. Controlled Release 126, 237-245 (2008).

8. Dumas, A., Moreau, M.F., Gherardi, R.K., Basle, M.F. \& Chappard, D. Bone grafts cultured with bone marrow stromal cells for the repair of critical bone defects: an experimental study in mice. $J$. Biomed. Mater. Res., Part A 90, 1218-1229 (2009).

9. Jia, W.T., Lau, G.Y., Huang, W.H., Zhang, C.Q., Tomsia, A.P. \& Fu, Q. Bioactive glass for large bone repair. Adv. Healthcare Mater. 4, 2842-2848 (2015).

10. Checchi, M., Bertacchini, J., Grisendi, G., Smargiassi, A., Sola, A., Messori, M. \& Palumbo, C. Proposal of a novel natural biomaterial, the scleral ossicle, for the development of vascularized bone tissue in vitro. Biomedicines 6, (2017).

11. Datta, P. Dhawan, A., Yu, Y., Hayes, D., Gudapati, H. \& Ozbolat, I.T. Bioprinting of osteochondral tissues: A perspective on current gaps and future trends. Int. J. Bioprint. 3, 109-120 (2017).

12. Dimitriou, R., Jones, E., McGonagle, D. \& Giannoudis, P.V. Bone regeneration: current concepts and future directions. BMC Med. 9, 66 (2011).

13. Maroulakos, M., Kamperos, G., Tayebi, L., Halazonetis, D. \& Ren, Y.J. Applications of 3D printing on craniofacial bone repair: A systematic review. $J$. Dent. 80, 1-14 (2019).

14. Santos, D., Silva, D.M., Gomes, P.S., Fernandes, M.H., Santos, J.D. \& Sencadas, V. Multifunctional PLLA-ceramic fiber membranes for bone regeneration applications. J. Colloid Interface Sci. 504, 101-110 (2017).

15. Agrawal, V. \& Sinha, M. A review on carrier systems for bone morphogenetic protein-2. J. Biomed. Mater. Res., Part B 105, 904-925 (2017).

16. Orellana, B.R., Thomas, M.V., Dziubla, T.D., Shah, N.M., Hilt, J.Z. \& Puleo, D.A. Bioerodible calcium sulfate/poly(beta-amino ester) hydrogel composites. J. Mech. Behav. Biomed. Mater. 26, 43-53 (2013).

17. Oryan, A., Alidadi, S., Moshiri, A. \& Maffulli, N. Bone regenerative medicine: classic options, novel strategies, and future directions. J. Orthop. Surg. Res. 9, 18 (2014).
18. Weng, L., Boda, S.K., Wang, H., Teusink, M.J., Shuler, F.D. \& Xie, J. Novel 3D hybrid nanofiber aerogels coupled with BMP-2 peptides for cranial bone regeneration. Adv. Healthcare Mater. 7, e1701415 (2018).

19. Wozney, J.M. \& Rosen, V. Bone morphogenetic protein and bone morphogenetic protein gene family in bone formation and repair. Clin. Orthop. Relat. Res. 346, 26-37 (1998).

20. Chen, B., Lin, H., Wang, J., Zhao, Y., Wang, B., Zhao, W., Sun, W. \& Dai, J. Homogeneous osteogenesis and bone regeneration by demineralized bone matrix loading with collagen-targeting bone morphogenetic protein-2. Biomaterials 28, 10271035 (2007).

21. Raftery, R.M., Mencia-Castano, I., Sperger, S., Chen, G., Cavanagh, B., Feichtinger, G.A., Redl, H., Hacobian, A. \& O'Brien, F.J. Delivery of the improved BMP-2-Advanced plasmid DNA within a gene-activated scaffold accelerates mesenchymal stem cell osteogenesis and critical size defect repair. J. Controlled Release 283, 20-31 (2018).

22. Kim, B.B., Kim, M., Park, Y.H. \& Park, J.B. Dexamethasone leads to upregulation of BMP6 and ACHE suppression of SMAD3 and ESR1 genes in human mesenchymal stem cells. Biochip J. 12, 222-230 (2018).

23. Shi, P., Chen, K. \& Goh, J.C. Efficacy of BMP-2 delivery from natural protein based polymeric particles. Adv. Healthcare Mater. 2, 934-939 (2013).

24. Kim, S.S., Gwak, S.J. \& Kim, B.S. Orthotopic bone formation by implantation of apatite-coated poly(lactide-co-glycolide)/hydroxyapatite composite particulates and bone morphogenetic protein-2. J. Biomed. Mater. Res., Part A 87, 245-253 (2008).

25. Bauer, T.W. \& Muschler, G.F. Bone graft materials. An overview of the basic science. Clin. Orthop. Relat. Res. 371, 10-27 (2000).

26. Yu, X., Khalil, A., Dang, P.N., Alsberg, E. \& Murphy, W.L. Multilayered inorganic microparticles for tunable dual growth factor delivery. $A d v$. Funct. Mater. 24, 3082-3093 (2014).

27. Metzger, S. Lienemann, P.S., Ghayor, C., Weber, W., Martin, I., Weber, F.E. \& Ehrbar, M. Modular poly(ethylene glycol) matrices for the controlled 3D-localized osteogenic differentiation of mesenchymal stem cells. Adv. Healthcare Mater. 4, 550558 (2015).

28. Samorezov, J.E., Headley, E.B., Everett, C.R. \& Alsberg, E. Sustained presentation of BMP-2 enhances osteogenic differentiation of human adiposederived stem cells in gelatin hydrogels. J. Biomed. Mater. Res., Part A 104, 1387-1397 (2016).

29. Seo, B.B., Choi, H., Koh, J.T. \& Song, S.C. Sustained BMP-2 delivery and injectable bone regen- 
eration using thermosensitive polymeric nanoparticle hydrogel bearing dual interactions with BMP-2. $J$. Controlled Release 209, 67-76 (2015).

30. Ma, C., Chang, B., Jing, Y., Kim, H. \& Liu, X. Bio-inspired micropatterned platforms recapitulate 3D physiological morphologies of bone and dentinal cells. Adv. Sci. (Weinh) 5, 1801037 (2018).

31. Zhu, L., Luo, D. \& Liu, Y. Effect of the nano/ microscale structure of biomaterial scaffolds on bone regeneration. Int. J. Oral Sci. 12, 6 (2020).

32. Yoon, S. Chang, J., Kwon, N., Moon, S., Park, Y., Han, K.H., Lim, B. \& Lee, J.H. Multifunctional nanomaterial-alginate drug delivery and imaging system for cancer therapy. Biochip J. 13, 236-242 (2019).

33. Ramon-Azcon, J., Ahadian, S., Obregon, R., Camci-Unal, G., Ostrovidov, S., Hosseini, V., Kaji, H., Ino, K., Shiku, H., Khademhosseini, A. \& Matsue, T. Gelatin methacrylate as a promising hydrogel for 3D microscale organization and proliferation of dielectrophoretically patterned cells. Lab Chip 12, 2959-2969 (2012).

34. Kim, J.M., Kim, W.J., Kim, M.Y., Kim, K.P., Sim, S.J. \& Kim, S.K. Development of hydrogel microparticle based RT-qPCR for advanced detection of BCR-ABL1 transcripts. Biochip J. 13, 182-190 (2019).

35. Wu, B. Takeshita, N., Wu, Y., Vijayavenkataraman, S., Ho, K.Y., Lu, W.F. \& Fuh, J.Y.H. Pluronic F127 blended polycaprolactone scaffolds via e-jetting for esophageal tissue engineering. $J . M a$ ter. Sci.: Mater. Med. 29, 140 (2018).

36. Chen, Y.C., Lin, R.Z., Qi, H., Yang, Y., Bae, H., Melero-Martin, J.M. \& Khademhosseini, A. Functional human vascular network generated in photocrosslinkable gelatin methacrylate hydrogels. $A d v$. Funct. Mater. 22, 2027-2039 (2012).

37. Wang, H., Zhou, L., Liao, J., Tan, Y., Ouyang, K., Ning, C., Ni, G. \& Tan, G. Cell-laden photocrosslinked GelMA-DexMA copolymer hydrogels with tunable mechanical properties for tissue engineering. J. Mater. Sci.: Mater. Med. 25, 2173-2183 (2014).

38. Li, L., Yan, B., Yang, J., Chen, L. \& Zeng, H. Novel mussel-inspired injectable self-healing hydrogel with anti-biofouling property. Adv. Mater. 27, 1294-1299 (2015).

39. Li, Y., Thambi, T. \& Lee, D.S. Co-delivery of drugs and dgnes using polymeric nanoparticles for synergistic cancer therapeutic effects. Adv. Healthcare Mater. 7, 1700886 (2018).

40. Zelikin, A.N., Price, A.D. \& Stadler, B. Poly (methacrylic acid) polymer hydrogel capsules: drug carriers, sub-compartmentalized microreactors, artificial organelles. Small 6, 2201-2207 (2010).
41. Ahmed, E.M. Hydrogel: Preparation, characterization, and applications: A review. J. Adv. Res. 6, 105-121, (2015).

42. Serra, L., Domenech, J. \& Peppas, N.A. Drug transport mechanisms and release kinetics from molecularly designed poly(acrylic acid-g-ethylene glycol) hydrogels. Biomaterials 27, 5440-5451 (2006).

43. Bertz, A., Wohl-Bruhn, S., Miethe, S., Tiersch, B., Koetz, J., Hust, M., Bunjes, H. \& Menzel, H. Encapsulation of proteins in hydrogel carrier systems for controlled drug delivery: influence of network structure and drug size on release rate. J. Biotechnol. 163, 243-249 (2013).

44. Coombes, A.G., Rizzi, S.C., Williamson, M., Barralet, J.E., Downes, S. \& Wallace, W. A. Precipitation casting of polycaprolactone for applications in tissue engineering and drug delivery. Biomaterials 25, 315-325 (2004).

45. Koutsopoulos, S. \& Zhang, S. Two-layered injectable self-assembling peptide scaffold hydrogels for long-term sustained release of human antibodies. $J$ Control Release 160, 451-458, (2012).

46. Woo, K.M., Chen, V.J. \& Ma, P.X. Nano-fibrous scaffolding architecture selectively enhances protein adsorption contributing to cell attachment. $J$. Biomed. Mater. Res., Part A 67, 531-537 (2003).

47. Alamein, M.A., Liu, Q., Stephens, S., Skabo, S., Warnke, F., Bourke, R., Heiner, P. \& Warnke, P.H. Nanospiderwebs: artificial 3D extracellular matrix from nanofibers by novel clinical grade electrospinning for stem cell delivery. Adv. Healthcare Mater. 2, $702-717$ (2013).

48. Macdonald, M. L. et al. Tissue integration of growth factor-eluting layer-by-layer polyelectrolyte multilayer coated implants. Biomaterials 32, 1446-1453, (2011).

49. Dimitriou, R., Mataliotakis, G.I., Calori, G.M. \& Giannoudis, P.V. The role of barrier membranes for guided bone regeneration and restoration of large bone defects: current experimental and clinical evidence. BMC Med. 10, 81 (2012).

50. Vo, T.N., Kasper, F.K. \& Mikos, A.G. Strategies for controlled delivery of growth factors and cells for bone regeneration. Adv. Drug Delivery Rev. 64, 1292-1309 (2012).

51. Lee, D.H., Park, J.Y., Lee, E.J., Choi, Y.Y., Kwon, G.H., Kim, B.M. \& Lee, S.H. Fabrication of three-dimensional microarray structures by controlling the thickness and elasticity of poly (dimethylsiloxane) membrane. Biomed. Microdevices 12, 49-54 (2010).

52. Lee, K.H., No, D.Y., Kim, S.H., Ryoo, J.H., Wong, S.F. \& Lee, S.H. Diffusion-mediated in situ alginate encapsulation of cell spheroids using mi- 
croscale concave well and nanoporous membrane. Lab Chip 11, 1168-1173 (2011).

53. Bertassoni, L.E., Cecconi, M., Manoharan, V., Nikkhah, M., Hjortnaes, J., Cristino, A.L., Barabaschi, G., Demarchi, D., Dokmeci, M.R., Yang, Y.Z. \& Khademhosseini, A. Hydrogel bioprinted microchannel networks for vascularization of tissue engineering constructs. Lab Chip 14, 22022211 (2014).

54. Roh, D., Choi, W., Kim, J., Yu, H.Y., Choi, N. \&
Cho, I.J. Fabrication of multi-layered macroscopic hydrogel scaffold composed of multiple components by precise control of UV energy. Biochip $J$. 12, 280-286 (2018).

55. Kim, M.J., Lee, B., Yang, K., Park, J., Jeon, S., Um, S.H., Kim, D.I., Im, S.G. \& Cho, S.W. BMP-2 peptide-functionalized nanopatterned substrates for enhanced osteogenic differentiation of human mesenchymal stem cells. Biomaterials 34, 72367246 (2013). 